\title{
Cost-Effectiveness of Therapeutic Education to Prevent the Development and Progression of Type 2 Diabetes: Systematic Review
}

\author{
Irina Odnoletkova1, Geert Goderis ${ }^{1}$, Lore Pil ${ }^{2}$, Frank Nobels ${ }^{3}$, Bert Aertgeerts ${ }^{1}$, Lieven Annemans ${ }^{4}$ and Dirk Ramaekers ${ }^{1}$ \\ ${ }^{1}$ Department of Public Health and Primary Care, University of Leuven, Belgium \\ ${ }^{2}$ Ghent University, Belgium \\ ${ }^{3} \mathrm{OLV}$ Hospital Aalst, Belgium \\ ${ }^{4}$ Ghent University \& Brussels University, Belgium
}

\begin{abstract}
Objective: To update current evidence on the cost-effectiveness (CE) of therapeutic education in prediabetes and type 2 diabetes.

Research design and methods: A systematic review of economic evaluations of therapeutic education in prediabetes and type 2 diabetes, based on Randomized Controlled Trials (RCTs) and published in $2002-2014$. The quality of the clinical evidence was appraised through the Cochrane Collaboration's tool for assessing risk of bias. Economic studies were evaluated through the Consensus Health Economic Criteria List. The Incremental CostEffectiveness Ratios (ICERs) of patient education in prediabetes and type 2 diabetes were compared.

Results: Out of 2031 identified publications, eight studies on prediabetes and nine on type 2 diabetes met the inclusion criteria. The level of the underlying clinical evidence was overall high in studies on prediabetes and varied in studies on type 2 diabetes. The mean ICER $(95 \% \mathrm{Cl})$ from the perspective of the healthcare system was $€ 18,000$ per QALY (range from dominance to $€ 49,700$ ) in prediabetes and $€ 29,700$ (range from $€ 9,100$ to $€ 50,300$ ) per QALY in type 2 diabetes. General flaws in the economic evaluations were short time horizons, limited uncertainty analysis and a lack of transparency in the modeling methods.

Conclusions: The number of economic evaluations of patient education in prediabetes and type 2 diabetes has been growing in the past years. Our review compares the health economic evidence on therapeutic education for both conditions. The findings suggest that offering therapeutic education already in prediabetes stage may be a better value for money than postponing it till after the diagnosis. More robust methodologies in health economic evaluations are essential in further evidence generation.
\end{abstract}

Keywords: Therapeutic education; Prediabetes; Type 2 diabetes; Economic evaluations; Cost-effectiveness

\section{Introduction}

Diabetes mellitus is a chronic illness that requires continuing medical care and ongoing patient self-management education [1]. About 382 million people worldwide have diabetes and its prevalence is expected to increase by more than $50 \%$ in the coming twenty years [2]. The associated healthcare costs exceeded USD 548 billion in 2013 [2]. About $90 \%$ of the diabetes population suffers from type 2 diabetes. Persons at high risk of type 2 diabetes usually progress slowly from the onset to the actual diagnosis during a period of eight to twelve years without experiencing any specific symptoms [3]. High risk of type 2 diabetes has been referred to as prediabetes in people with Impaired Fasting Glucose (IFG) and/ Or Impaired Glucose Tolerance (IGT) [1]. Reduction in hyperglycemia, hypertension, and cardiovascular risk factors in people with prediabetes and type 2 diabetes is believed to reduce the risk of future micro- and macrovascular complications [4]. Early detection of both conditions and patient support in selfmanagement of their risk factors should be of crucial importance for the public health care policies.

Therapeutic patient education (also referred to as patient - or selfmanagement education in this article) is an integral part of treatment and is defined as a collaborative process through which people with or at risk of type 2 diabetes gain the knowledge and skills needed to modify behavior and better manage their diabetes risk factors $[5,6]$. Multiple systematic reviews concluded that patient education is effective in improving glycemic control in people with type 2 diabetes in the short term [7-9].
In individuals with prediabetes, a reduction in the rate of conversion to type 2 diabetes after intensive lifestyle interventions was demonstrated in several well-conducted randomized controlled trials, with risk reduction of $51 \%$ to $58 \%$ compared to controls in the short term and sustained risk reduction of $34 \%$ to $43 \%$ over a follow-up period of between 7 and 20 years [10]. Reversion to normal glucose regulation, even if transient, is associated with a significantly reduced risk of future diabetes [11]. In the above-mentioned studies, patient education was the fundamental component of the lifestyle interventions, with the aim of persuading people about an evidence-based diet and physical activity management.

Current health economic evidence on the topic is too limited to formulate unambiguous recommendations on reimbursement policies. Several reviews of the Cost-Effectiveness (CE) of patient education in people with type 2 diabetes have been undertaken, without generating

*Corresponding author: Irina Odnoletkova, University of Leuven, Kapucijnenvoer 33, Leuven, B-3000, Tel: 0032 (0) 473368010; Fax: 0032 (0) 27789400 ; E-mail: irina.odnoletkova@mloz.be

Received August 30, 2014; Accepted September 22, 2014; Published October 02, 2014

Citation: Odnoletkova I, Goderis G, Pil L, Nobels F, Aertgeerts B, et al. (2014) Cost-Effectiveness of Therapeutic Education to Prevent the Development and Progression of Type 2 Diabetes: Systematic Review. J Diabetes Metab 5: 438 doi:10.4172/2155-6156.1000438

Copyright: ( 2014 Odnoletkova I, et al. This is an open-access article distributed under the terms of the Creative Commons Attribution License, which permits unrestricted use, distribution, and reproduction in any medium, provided the original author and source are credited. 
any clear conclusions, mostly due to scarcity of publications or the limited quality of the studies [12-15]. Most economic evaluations of educational and lifestyle interventions to prevent type 2 diabetes in people with IGT were based on the results of the Diabetes Prevention Program [16]. Application of different modeling techniques produced conflicting results: in general very cost-effective [17-19], but not costeffective in the study by Eddy et al. [20].

The objective of this review is to update the knowledge on CE of therapeutic educational programs for people with prediabetes and type 2 diabetes. This study will be the first to compare the existing evidence on both conditions.

\section{Methods}

\section{Data sources and searches}

To identify the relevant studies we searched the Medical Literature Analysis and Retrieval System Online (MEDLINE), Excerpta Medica (EMBASE), Cumulative Index to Nursing and Allied Health Literature (CINAHL), the Cochrane library, the Centre for Reviews and Dissemination database (CRD), Econlit. The search strategy was based on the combination of the following terms and their proximate notions: 1) type 2 diabetes or prediabetes; 2) patient education (including tele-counseling); and 3) costs or economics or quality adjusted life years or modeling.

\section{Study selection}

Criteria for inclusion in the review were a combination of the following study characteristics: 1) the study population are adults with diagnosed type 2 diabetes or prediabetes; 2 ) the intervention is any structural program whose purpose is to improve disease knowledge and self-management skills, performed by any type of caregiver, with the use of any supporting material or devices; 3 ) the comparator is usual care; 4) the economic evaluation is based on a Randomized Controlled Trial (RCT); 5) the health effects are measured as Quality-Adjusted Life Years Gained (QALYs) or Life Years Gained (LYs); 6) the Incremental Cost-Effectiveness Ratio (ICER) is reported unless the intervention is dominant or dominated ; and 7) the study was published between January 2002 and April 2014.

Exclusion criteria were: 1) partial economic evaluation, i.e. no analysis of the incremental costs of the intervention in relation to the incremental treatment effects; 2) economic modeling studies based on systematic reviews or meta-analyses, - due to variability in the organization of behavioral interventions and their effectiveness; 3 ) educational intervention did not include a human interaction, e.g. print - or video material; 4) the study participants represent a particular subgroup of patients with type 2 diabetes or prediabetes potentially limiting the generalizability of the results; and 5) published before 2002. Earlier publications were not expected to apply the established methodologies for cost-effectiveness analysis as demonstrated by the previous systematic reviews [13-15].

The study selection was performed by two reviewers independently (IO and GG) in three rounds: titles, abstracts, full text. To reduce the risk of omission, the studies were included in the following selection round if they were selected by at least one of the reviewers. In the last round - full text inclusion - the study supervisors assisted in reaching a consensus on the final selection of the studies. The inter-rater agreement was calculated by means of kappa statistics after each selection round.

\section{Data extraction and quality of evidence assessment}

After the final selection of the economic evaluations, the original clinical studies were retrieved. To assess the quality of the health economic evidence, we first evaluated the internal validity of the RCTs by using the Cochrane Collaboration's tool for assessing risk of bias and then applied the GRADE methodology to rank the level of the clinical evidence. High, moderate, low or very low grade of evidence was assigned to the studies with downgrading each time when one of the following was suggested: 1) limitations in design and implementation based on each topic of the risk of bias analysis; 2) indirectness of evidence; 3) unexplained heterogeneity or inconsistency of results; 4) imprecision of results; and 5) high probability of publication bias [21] IO and GG performed the assessment independently.

To critically appraise the quality of the economic evaluations, the Consensus Health Economic Criteria List (CHEC) was used [22]. We have extended the CHEC with a brief guidance to support each value judgment based on recently published methodologies and one question, specifically applicable to the decision-analytic models [23-25]. The following data were extracted for each study: the target population, the type of intervention, the comparator and the effectiveness results observed in the original RCT; the analytic horizon and the study perspective; the health and economic consequences of the alternative treatments considered and methods to measure and value costs and health outcomes; the discounting methods; and the performed uncertainty analysis. For the modeling studies, the models' structural assumptions and validation methods were extracted. Each question of CHEC was answered with "yes/rather yes", "no/rather no", or "unclear" and justified. IO and LP assessed the studies independently and agreed on the assessment results.

\section{Data Synthesis and Analysis}

We reported the review results by grouping the economic evaluations per target population, based on the cost-effectiveness of the education program - from most to least cost-effective. For each study, the target population, the type of intervention, and the effectiveness results are reported; and the analytic horizon and the study perspective are specified. If not clearly stated in the study, the perspective was assumed based on the types of costs included in the analysis and reported as "assumed". ICERs were rounded up to hundreds. To make ICERs comparable across the studies we converted all currencies into Euros by using the average standardized exchange rate of 2012 [26]. If the cost valuation year was not explicitly mentioned, we have assumed it to be the year prior to the study publication. For the purpose of the descriptive statistics, interventions which were found cost-saving, were included into the ICER analysis with the ICER value equal to zero. We adopted the classification of CE applied by Li et al. [17] as established by convention: dominant, or cost-saving; or - depending on costs per QALY - very cost-effective $(0<$ ICER $\leq € 20,000)$; cost-effective $(€ 20,000<$ ICER $\leq € 40,000)$; marginally cost-effective $(€ 40,000<$ ICER $\leq € 80,000)$; not cost-effective ( $>€ 80,000)$.

The methodological quality of the studies was summarized by using the Review Manager software.

\section{Results}

\section{Design and quality of the included studies}

The search yielded 2031 publications. Seventeen studies met the inclusion criteria; eight of them evaluated CE of lifestyle interventions in prediabetes. The study selection process is depicted in Figure 1. Eleven studies were based on decision analytic models and considered a longterm time horizon. All models with the exception of that used by Eddy et al. [20] were Markov state transitions models in which participants 


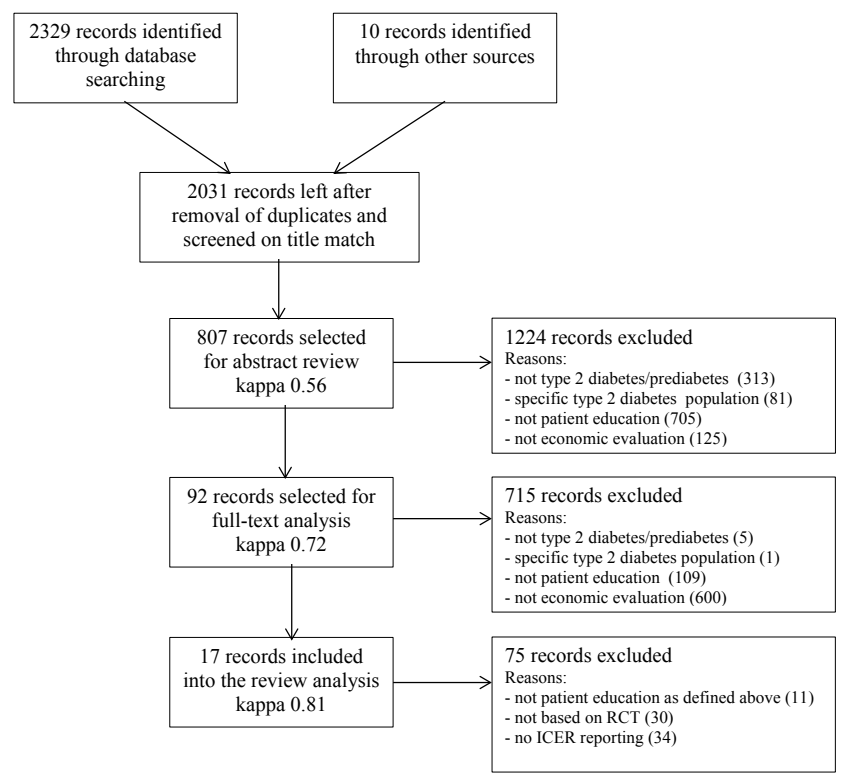

Figure 1: Selection of cost-effectiveness studies for systematic review of therapeutic education to prevent the development and progression of type 2 diabetes.

move from and to defined health states within discrete time periods. Most studies on prediabetes included the healthcare system and the societal perspective. The societal perspective was not considered in any of the studies on type 2 diabetes.

The level of clinical evidence from the original clinical trials was ranked high in $50 \%$ of the RCTs and was overall higher in studies on prediabetes. One study on prediabetes and three studies on type 2 diabetes were within-trial economic evaluations with a time horizon of no more than 3 years and as such ignored the long-term consequences of the interventions with regard to health outcomes and the associated treatment costs. Most modeling studies failed to deliver a transparent presentation of the model structural assumptions and validation methods. None of the studies was exhaustive in the performance and presentation of the uncertainty analysis. The accuracy in identification, measurement and valuation of the costs and outcomes varied. Outcomes of the reporting and quality assessment of the economic evaluations based on CHEC are summarized in Figure 2. A detailed report on the methodological flaws of the RCTs and the economic evaluations is available upon author's request.

\section{Cost-effectiveness of the studied interventions}

The mean ICER (95\% CI) from the perspective of the healthcare system was $€ 18,000$ per QALY gained (range from dominant to $€ 49,700$ ) in prediabetes and $€ 29,700$ (range from $€ 9,100$ to $€ 50,300$ ) in type 2 diabetes. The boxplots of ICERs are presented in Figure 3.

Below, we give a brief description of each study by reporting results from the original RCT, the type of intervention, the analytic technique applied and the CE results.

\section{Prediabetes}

From eight studies on prediabetes, four were based on the Diabetes Prevention Program (DPP) [16] and three on 10 years follow-up of DPP. DPP is a well conducted RCT which demonstrated $58 \%$ reduction in incidence of type 2 diabetes in the intensive lifestyle group and $31 \%$ in the metformin group compared to the placebo group at 2.8 years follow-up. The educational intervention was an intensive lifestylemodification program with the goals of at least a $7 \%$ weight loss and at least 150 minutes of physical activity per week offered in a curriculum of 16 individual lessons during the first 6 months and subsequent individual and group lessons, mostly on a monthly basis.

Six out of eight studies found intensive lifestyle interventions cost-saving or very cost-effective: Palmer et al. [27] used the results of DPP to analyze the CE in five countries - Australia, France, Germany, Switzerland and the UK, - by applying a simple three-state Markov
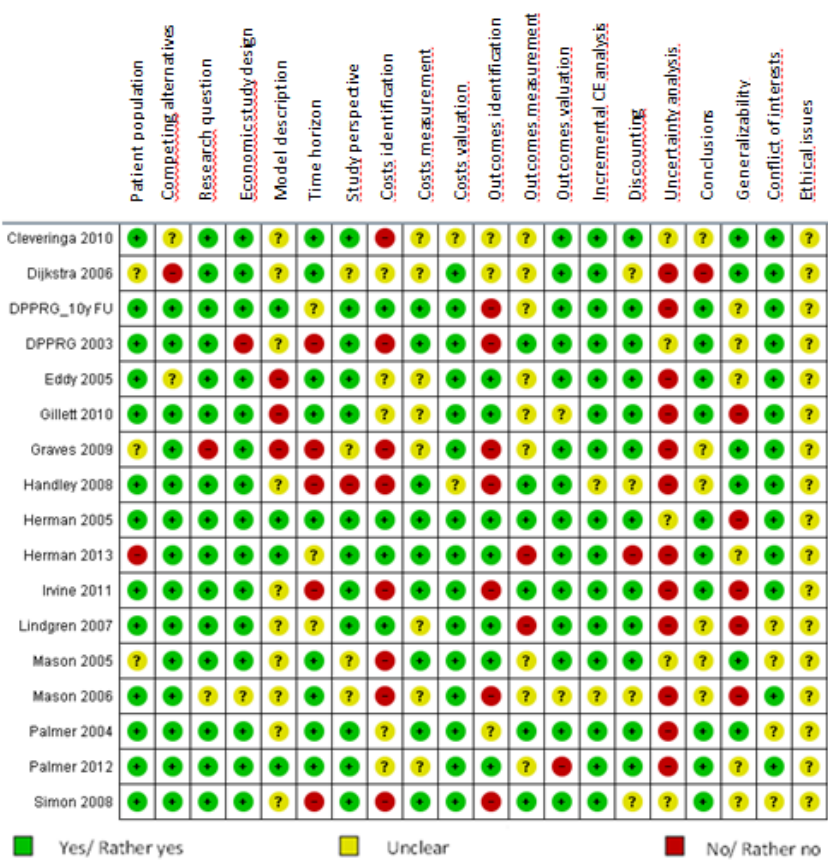

Figure 2. Authors' judgments about compliance with good practices in the included studies, for each item of the modified CHEC checklist.

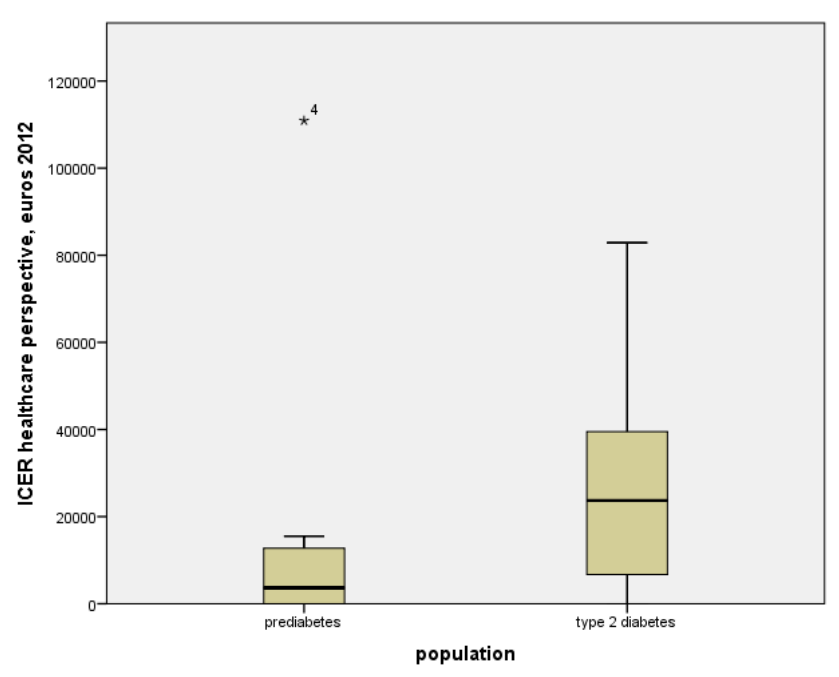

Figure 3: Boxplots of the incremental cost-effectiveness ratios in Euros (2012) per QALY gained of patient education in prediabetes and type 2 diabetes from the perspective of the healthcare system. 
model with the states IGT, alive with type 2 diabetes and deceased, over a lifetime horizon. The intervention was found to be cost-saving in all countries except in the UK, where it was very cost-effective with an ICER of $€ 6400$ per life-year gained.

Lindgren et al. [28] investigated lifelong CE based on the Finnish Diabetes Prevention Study [29,30]. This RCT of good quality demonstrated a $58 \%$ reduction in cumulative incidence of type 2 diabetes at 6 years compared to controls. The intervention consisted of individual nutritionist advice on reduction in weight of at least $5 \%$, total fat intake less than $30 \%$ and saturated fat intake less than $10 \%$, fiber intake of $15 \mathrm{~g} / 1000 \mathrm{kcal}$, and moderate exercise of at least 30 minutes per day. In addition, supervised individual resistance training to improve the strength of the large muscle groups was offered. In the long-term effect analysis, the transition states of the used model are limited to IGT, type 2 diabetes, myocardial infarction/ stroke and death. The lifestyle intervention was found to be cost-saving from the payers and the societal perspective with a maximal cost of $€ 2400$ per QALY gained.

Palmer et al. [31] projects lifetime clinical and economic outcomes based on the results from the DPP and a 10 years follow-up of DPP, Diabetes Prevention Program Outcomes Study (DPPOS), from a third-party payer perspective in Australia [32]. The model is similar to that applied in Palmer et al. [27] but adds one additional health state, - "normal glucose regulation" and thus accounts for reversion to normoglycemia. Intensive lifestyle intervention comes out as a costsaving intervention, metformin costs AUD10100 (€8100) per QALY.

Herman et al. [33] explored the CE of DPP through lifetime modeling. Probabilities of transition from IGT to diabetes onset, type 2 diabetes, all known diabetes complications and death were included in the analysis based on an existing model, built mainly on data from the United Kingdom Prospective Diabetes Study (UKPDS). The ICER of the intensive lifestyle intervention was $\$ 1100$ (€900) per QALY from the healthcare perspective and $\$ 8,800$ (€6800) per QALY from the societal perspective [34].

"DPPRG, 10 years follow-up", 2012, U.S. [35] is a within-trial CE analysis of DPPOS [36]. At 10 years, the incidence of diabetes was $34 \%$ and $18 \%$ lower in the intensive lifestyle and the metformin group respectively if compared to the placebo group. After DPP, all groups were offered 16 lifestyle support group sessions during 7 months (DPPbridge). In the 5.7 subsequent years, all groups could participate in the Healthy Lifestyle Program (HELP) consisting of four quarterly onehour group visits. In addition, the original intensive lifestyle group was offered two group classes aimed at self-management behaviors for weight loss (BOOST). The metformin group continued with metformin. The attendance rate was $18 \%$ for HELP and $17 \%$ for BOOST. The ICER was $\$ 12900$ (€10000) per QALY from the healthcare system perspective and $\$ 19800(€ 15300)$ from the societal perspective.

Herman et al. [36] is based on DPPRG, 10 years follow-up and is a post-hoc CE analysis for the subgroup of "adherent" patients. Adherent lifestyle participants were defined as those without diabetes who achieved and maintained 5\% weight loss at $\geq 50 \%$ of their semi-annual visits; adherent metformin participants as those without diabetes who took $\geq 80 \%$ of their prescribed metformin; adherent placebo participants as all those randomized to the placebo group. The incidence of diabetes among "adherent" patients from the intensive lifestyle group at 10 years was $49.4 \%$ lower than in placebo and $20.8 \%$ lower than in metformin group. For the intensive lifestyle intervention, ICERs were $\$ 20,000$ $(€ 15,500)$ and $\$ 3200(€ 2500)$ per QALY from the healthcare and the societal perspective respectively. Metformin costs $\$ 20200(€ 15,700)$ per
QALY, from the healthcare system perspective, and is cost-saving from the societal perspective. In this study, the measure of adherence was the treatment effect in the intensive lifestyle group and the compliance to the intervention protocol in the metformin group, while in the placebo group all patients were considered adherent. The inconsistency in the definition of adherence across groups seems a substantial flaw of the study design and its results should be interpreted with caution.

Marginal cost-effectiveness was found in the within-trial economic evaluation along DPP, which provides an accurate reporting of costs and outcomes within the RCT [37]. However, due to the short time horizon, it ignores all future consequences of the alternative treatments. The ICER of $\$ 51600(€ 40,000)$ per QALY gained, calculated from the societal perspective, should therefore be interpreted with caution. Intensive lifestyle intervention was not cost-effective in the study of Eddy et al. [20] which explored the lifetime CE of DPP by using the Archimedes Diabetes model [38]. The model is based on objectoriented programming and does not involve fixed health transition states. It consists of hundreds of variables that interact within hundreds of equations. The model was extensively validated and is described as highly precise. Eddy et al. conclude that delaying the lifestyle intervention until after a person develops diabetes would be more costeffective than offering it to people with IGT. The reported ICERs were $\$ 1,43,000(€ 1,10,900)$ per QALY from the health plan's perspective and $\$ 62,600(€ 48,500)$ per QALY from the societal perspective.

\section{Type 2 diabetes}

In type 2 diabetes, therapeutic education was found cost-saving only in the study of Mason et al. [39], and only for the subgroup of people with hypertension where the intervention was focused on blood pressure lowering strategies in the UK. It was very cost-effective in the same study for the subgroup of people with dyslipidemia with an ICER of $\$ 8230$ (€6400) per QALY gained, supposedly from the health care perspective. The underlying RCT is of a good quality and demonstrates a significant increase of the percentage of patients at target for the level of total cholesterol (but not for the level of blood pressure) [40]. The specialist nurse-led intervention in this study was aimed at better control of hypertension and hyperlipidemia in diabetes and included individual target-driven lifestyle counselling and medication adjustment. The applied model includes the states: type 2 diabetes, myocardial infarction, stroke and death.

Diabetes education was very cost-effective also in two other studies: Gillett et al. [41] investigated the lifetime CE of education for ongoing and newly diagnosed diabetics in the UK and found an ICER of $£ 5387$ ( $€ 6700$ ) per QALY, from the healthcare system perspective. The one-year intervention was a structured 6-hours group education program focused on lifestyle and goal setting and delivered by professional health educators. There was no significant betweengroup difference in effect on $\mathrm{HbAlc}$, but there was a positive significant effect in odds of non-smoking [42]. The study used the Sheffield type 2 diabetes models that include progression from diabetes without complications to micro- and macrovascular complications.

Dijkstra et al. [43] analyzed the life-long CE of a diabetes passport - an intervention that included education sessions for patients and professionals in hospital setting [44]. Difference in HbA1c change after one year was $0.5 \%$ and statistically significant. The NIDDM model was used for the long-term extrapolation of results. Though the original clinical study reports a 2 -arm design with a single complex intervention, ICERs of $€ 16400$ per QALY are reported for the patient-centric and $€ 32200$ per QALY for the provider-centric intervention. 
Educational interventions in type 2 diabetes were cost-effective in three studies: Graves et al. [45] analyzed the CE of telephone counseling for physical activity and diet in primary care patients with type 2 diabetes or hypertension [46]. The RCT was of a good quality and demonstrated a significant improvement in intake of fat, vegetables, fruit and fiber achieved through 18 lifestyle telephone sessions offered by nutritionists. The model applies a 10 -year time horizon and transition to different behavior states (suboptimal lifestyle - improved diet - improved exercise - improved diet and exercise) or death, each assigned a particular QALY value. The model is non-conventional as it uses health behaviors instead of health states. It is not clear how the evolution of the health status was incorporated into the model, nor how the transition probabilities and the associated health utility values were estimated beyond the trial. ICERs were AUD78500 $(€ 63,300)$ per QALY compared to usual care and AUD29400 (€23,700) per QALY compared to "real controls".

Cleveringa et al. [47] investigated the life-long CE of the Diabetes Care Protocol, a complex one-year intervention that included patient education and medication prescription by nurses upon approval of GPs and with support of a computerized decision support system [48]. This well conducted RCT showed no significant difference in effect on HbA1c but did in the secondary outcomes - blood pressure and lipid profile. The model is based on Eastman's Model of complications of Non-Insulin Dependent Diabetes Mellitus (NIDDM) adapted to the Dutch population and simulates progression to Cardiovascular Diseases (CVD) - angina pectoris and myocardial infarction, microvascular complications and death [49]. An ICER of $€ 38200$ per QALY from the health care perspective was reported. Although, the clinical trial did not include the subgroup analysis for people with and without CVD, the economic evaluation does report the results for these subgroups: for patients with CVD, the ICER was $€ 14800$, and for patients without CVD $€ 121300$ per QALY.

The study of Handley et al. [50] is a within-trial economic evaluation of a nine-month education offered to patients with poorly controlled HbAlc. The intervention consisted of an automated telephone questionnaire and the outbound calls by nurse initiated when the answers are "out of range" [51]. Both the clinical and the economic evaluation contain some essential methodological flaws. The ICER ranged from \$29402 to \$72407 (€22800 - €56100) per QALY.

A marginal cost-effectiveness of $£ 43400$ ( $€ 53600$ ) per QALY gained was found in the study of Mason et al. [52] which explored the life-long CE of pro-active call center treatment support for people diagnosed with type 2 diabetes since more than one year in primary care [53]. The original well-conducted RCT demonstrates a statistically significant reduction of $\mathrm{HbAlc}$ after one year by $0.31 \%$, achieved through outbound phone self-management support counselling with tailored intensity delivered by diabetes nurses. The long-term effects simulated in the model were not reported. Only a change in HbAlc was considered a risk factor of the disease progression.

Educational support was not cost-effective in the study of Irvine et al. [54] which combines the effectiveness and the health economic analysis in one publication and investigates the CE of group education for people with IGT and newly diagnosed type 2 diabetes. The study has many methodological limitations. The reported ICER is $£ 67174$ (€82900) per QALY. More favorable results were found for the subgroups of people with IFG and those with a follow-up time longer than 4 months: $£ 20620$ (€25500) and $£ 17075$ (€21000) per QALY respectively.
The study of Simon et al. [55] is a within-trial economic evaluation. It was based on a 3-arm RCT in which usual care was compared to the Self-Monitoring of Blood Glucose (SMBG) and to the SMBG along with coaching for people with non-insulin treated type 2 diabetes [56]. The study reports low adherence rates: only 54 out of 151 people are adherent to the intensive SMBG; only 85 out of 150 - in the normal SMBG. No significant improvement in glycaemic control was found after one year. In both intervention groups, a non-significant loss in QALYs was observed. SMBG was evidently found to be dominated by usual care.

\section{Discussion}

The main finding of our review is that that therapeutic education may be a good value for money in patients with pre-diabetes and type 2 diabetes. Current evidence suggests that offering education programs already in prediabetes stage would be a better strategy than postponing them till after the diagnosis.

Six out of eight studies on prediabetes found patient education costsaving or very cost-effective. Other two studies reported less favorable results. One of them considered only a short-term analytic horizon. The meaningfulness of short-term cost-effectiveness analysis of therapeutic education in type 2 diabetes is questionable. As confirmed by 10 years within-trial analysis of DPP, the greatest costs are observed in the year of the delivery and decreased in the subsequent years, while most of the benefits occurred after 3 years of follow-up [35].

The other study was a modeling study by Eddy et al. [38] which concluded that intensive lifestyle interventions are not cost-effective with an ICER of $€ 110900$ per QALY gained. Five other modeling studies predicted such interventions to be cost-saving or very cost-effective with a maximum ICER of $€ 6400$ per QALY gained. The discussion on the predictive accuracy of different models stayed unresolved in absence of real life data. Only after the recent publication of the 10-years withintrial CE analysis of DPPOS that calculated an ICER of $€ 10000$ per QALY, it becomes clear that the study by Eddy et al. produced results least consistent with the real life observations. This may be explained by adoption of a number of specific structural model assumptions, such as that the intervention would last for life, or that people with type 2 diabetes who achieve $\mathrm{HbA1c}$ below 7\%, stay well-controlled for the rest of their life.

In type 2 diabetes, the results were mixed and varied from costsaving to not cost-effective, or even dominated. Three out of nine studies were within-trial economic evaluations and thus considered only a short-term analytic horizon. None of the studies on type 2 diabetes included a long-term analysis of costs and outcomes based on real life data. The quality of the underlying clinical evidence was overall stronger in studies on prediabetes. Thus, our review suggests that patient education in prediabetes stage is more cost-effective and supported by stronger evidence.

\section{The following relativizing thoughts should help to better interpret the results of the review}

The health economic evaluations are generally performed when interventions are clinically effective. This review should thus not be used to draw conclusions on the clinical effectiveness of therapeutic education, but on the cost-effectiveness of interventions with a positive health effect, to support the policy makers and health professionals in their decisions.

Most economic evaluations in prediabetes were based on the results 
Citation: Odnoletkova I, Goderis G, Pil L, Nobels F, Aertgeerts B, et al. (2014) Cost-Effectiveness of Therapeutic Education to Prevent the Development and Progression of Type 2 Diabetes: Systematic Review. J Diabetes Metab 5: 438 doi:10.4172/2155-6156.1000438

Page 6 of 7

of the Diabetes Prevention Program. In studies on type 2 diabetes, the quality of the clinical evidence varied. More research on the topic is needed, preferably performed in real-life settings, with special attention to the barriers and facilitators of successful changes in clinical practice.

In the future, it will be important to identify subgroups where therapeutic education is expected to be more effective. The extent of betacell dysfunction might, for instance, independently affect the disease progression. The 5.8-year follow-up of DPP showed that participants of the intensive lifestyle arm, who did not return to normoglycemia at least once during the trial, had a higher risk of progression to diabetes than the control group [11]. A risk reduction of $56 \%$ was found in those who returned to normal glucose regulation irrespective of the previous allocation to the intensive lifestyle or metformin arm [11]

The overall challenge of reviews in health economics is that inconsistencies in the conduct and reporting of the cost-effectiveness studies complicate a systematic comparison of the results, particularly when different time horizons are chosen, different modeling methods applied and the uncertainty around the structure, its parameters and the methodology is not sufficiently explored. Commonly accepted methodologies to perform and assess economic evaluations would lead to a generation of higher quality health economic evidence.

One should be cautious with the generalization of the costeffectiveness results or their transfer to other settings. Next to potential differences in clinical effect - e.g. due to divergences in the organization of usual care, differences in the absolute and relative cost consequences may occur. The cost-effectiveness classification adopted in our review is conditional and does not necessarily reflect the national reimbursement policies. It is known that applying an explicit ICER threshold is not typical for the reimbursement policies in most countries [57]. Moreover, in general, cost-effectiveness is not the only criterion in the reimbursement decisions. Severity of disease, size of the target population, budget impact, and availability of the treatment alternatives may play a role next to legal, ethical and organizational issues.

While keeping in mind these limitations to the synthesis and generalizability of results, our review suggests that re-consideration of public health priorities in the direction of earlier prevention of diabetes might be appropriate. This would imply more efficient screening methods for detecting people with prediabetes. Targeting people at high risk, such as hypertensive and obese patients, is believed to be cost-effective [58]. More insights into the evolution of blood glucose levels are needed to come up with an optimal re-test frequency.

\section{Conclusion}

Therapeutic education may be cost-effective in prediabetes and type 2 diabetes, but offers a better value for money when offered in prediabetes stage.

\section{Acknowledgments}

We thank Mr. Jens De Groot for his support in designing and testing the search strategies and Mr. Xavier Brenez for his endorsement of the research on behalf of the Independent Sickness Funds of Belgium.

Author's contribution - 10 wrote the first draft of the manuscript and takes responsibility for the contents of the article. GG and LP acted as second reviewers and assessors. FN and BA contributed to the protocol design and critically revised the paper. DR and LA supervised the study.

\section{References}

1. Standards of Medical Care in Diabetes (2013) American Diabetes Association.

2. IDF Diabetes Atlas (2013).
3. Harris MI, Klein R, Welborn TA, Knuiman MW (1992) Onset of NIDDM occurs at least 4-7 yr before clinical diagnosis. Diabetes Care 15: 815-819.

4. Implications of the United Kingdom Prospective Diabetes Study. American Diabetes Association. (2002) Diabetes Care January vol. 25 no. suppl 1 s28-s32

5. Haas L, Maryniuk M, Beck J, Cox CE, Duker P, et al. (2013) Standards Revision Task Force. National standards for diabetes self-management education and support. Diabetes Care. 36 Suppl 1:S100

6. Therapeutic Patient Education. Continuing Education Programmes for Health Care Providers in the Field of Prevention of Chronic Diseases Report of a WHO Working Group (1998) ISBN 9289012986

7. Norris SL, Lau J, Smith SJ, Schmid CH, Engelgau MM (2002) Self-management education for adults with type 2 diabetes: a meta-analysis of the effect on glycemic control. Diabetes Care 25: 1159-1171.

8. Gary TL, Genkinger JM, Guallar E, Peyrot M, Brancati FL (2003) Meta-analysis of randomized educational and behavioral interventions in type 2 diabetes. Diabetes Educ 29: 488-501.

9. Minet L, Møller S, Vach W, Wagner L, Henriksen JE (2010) Mediating the effect of self-care management intervention in type 2 diabetes: a meta-analysis of 47 randomised controlled trials. Patient Educ Couns 80: 29-41.

10. Gillett M, Royle P, Snaith A, Scotland G, Poobalan A, et al. (2012) Nonpharmacological interventions to reduce the risk of diabetes in people with impaired glucose regulation: a systematic review and economic evaluation. Health Technol Assess. 16: 1-236

11. Perreault L, Pan Q, Mather KJ, Watson KE, Hamman RF, et al. (2012) Diabetes Prevention Program Research Group. Effect of regression from prediabetes to normal glucose regulation on long-term reduction in diabetes risk: results from the Diabetes Prevention Program Outcomes Study. Lancet 379:2243-2251

12. Loveman E, Cave C, Green C, Royle P, Dunn N, et al. (2003) The clinical and cost-effectiveness of patient education models for diabetes: a systematic review and economic evaluation. Health Technol Assess 7: iii, 1-190.

13. Vijgen SM, Hoogendoorn M, Baan CA, de Wit GA, Limburg W, et al. (2006) Cost effectiveness of preventive interventions in type 2 diabetes mellitus: a systematic literature review. Pharmacoeconomics 24: 425-441.

14. Urbanski P, Wolf A, Herman WH (2008) Cost-effectiveness of diabetes education. J Am Diet Assoc 108: S6-11.

15. Boren SA, Fitzner KA, Panhalkar PS, Specker JE (2009) Costs and benefits associated with diabetes education: a review of the literature. Diabetes Educ 35: 72-96.

16. Knowler WC, Barrett-Connor E, Fowler SE, Hamman RF, Lachin JM, et al. (2002) Reduction in the incidence of type 2 diabetes with lifestyle intervention or metformin. N Engl J Med 346: 393-403.

17. Li R, Zhang P, Barker LE, Chowdhury FM, Zhang X (2010) Cost-effectiveness of interventions to prevent and control diabetes mellitus: a systematic review. Diabetes Care 33: 1872-1894.

18. Saha S, Gerdtham UG, Johansson P (2010) Economic evaluation of lifestyle interventions for preventing diabetes and cardiovascular diseases. Int J Environ Res Public Health 7: 3150-3195.

19. Gillett M, Royle P, Snaith A, Scotland G, Poobalan A, et al. (2012) Nonpharmacological interventions to reduce the risk of diabetes in people with impaired glucose regulation: a systematic review and economic evaluation. Health Technol Assess. 16:1-236

20. Eddy DM, Schlessinger L, Kahn R (2005) Clinical outcomes and costeffectiveness of strategies for managing people at high risk for diabetes. Ann Intern Med 143: 251-264

21. Higgins J.P.T, Green S (editors) (2011) Cochrane Handbook for Systematic Reviews of Interventions Version 5.1.0 [updated March 2011]. The Cochrane Collaboration.

22. Evers S, Goossens M, de Vet H, van Tulder M, Ament A (2005) Criteria list for assessment of methodological quality of economic evaluations: Consensus on Health Economic Criteria. Int J Technol Assess Health Care 21: 240-245.

23. Cleemput I, Neyt M, Van de Sande S, Thiry N. (2012) Belgian guidelines fo economic evaluations and budget impact analyses: second edition. Health Technology Assessment (HTA). Brussels: Belgian Health Care Knowledge Centre (KCE). KCE Report 183C. D/2012/10.273/54 
Citation: Odnoletkova I, Goderis G, Pil L, Nobels F, Aertgeerts B, et al. (2014) Cost-Effectiveness of Therapeutic Education to Prevent the Development and Progression of Type 2 Diabetes: Systematic Review. J Diabetes Metab 5: 438 doi:10.4172/2155-6156.1000438

24. Weinstein MC, O'Brien B, Hornberger J, Jackson J, Johannesson M, et al. (2003) ISPOR Task Force on Good Research Practices--Modeling Studies. Principles of good practice for decision analytic modeling in health-care evaluation: report of the ISPOR Task Force on Good Research PracticesModeling Studies. Value Health. 6:9-17

25. Caro JJ, Briggs AH, Siebert U, Kuntz KM (2012) ISPOR-SMDM Modeling Good Research Practices Task Force. Modeling good research practices--overview: a report of the ISPOR-SMDM Modeling Good Research Practices Task Force--1. Value Health. 15:796-803

26. http://sdw.ecb.europa.eu/

27. Palmer AJ, Roze S, Valentine WJ, Spinas GA, Shaw JE, et al. (2004) Intensive lifestyle changes or metformin in patients with impaired glucose tolerance: modelling the long-term health economic implications of the diabetes prevention program in Australia, France, Germany, Switzerland, and the United Kingdom. Clin Ther 26:304-321

28. Lindgren P, Lindström J, Tuomilehto J, Uusitupa M, Peltonen M, et al. (2007) Lifestyle intervention to prevent diabetes in men and women with impaired glucose tolerance is cost-effective. Int J Technol Assess Health Care 23: 177 183.

29. Tuomilehto J, Lindström J, Eriksson JG, Valle TT, Hämäläinen H, et al. (2001) Finnish Diabetes Prevention Study Group. Prevention of type 2 diabetes mellitus by changes in lifestyle among subjects with impaired glucose tolerance. N Engl J Med 344:1343-1350

30. Lindström J, Eriksson JG, Valle TT, Aunola S, Cepaitis Z, et al. (2003) Prevention of diabetes mellitus in subjects with impaired glucose tolerance in the Finnish Diabetes Prevention Study: results from a randomized clinical trial. J Am Soc Nephrol. 14:S108-S113

31. Palmer AJ, Tucker DM (2012) Cost and clinical implications of diabetes prevention in an Australian setting: a long-term modeling analysis. Prim Care Diabetes 6: 109-121.

32. Diabetes Prevention Program Research Group, Knowler WC, Fowler SE, Hamman RF, Christophi CA, et al. (2009) 10-year follow-up of diabetes incidence and weight loss in the Diabetes Prevention Program Outcomes Study. Lancet 374:1677-1686.

33. Herman WH, Hoerger TJ, Brandle M, Hicks K, Sorensen S, et al. (2005) The cost-effectiveness of lifestyle modification or metformin in preventing type 2 diabetes in adults with impaired glucose tolerance. Ann Intern Med 142: 323 332

34. CDC Diabetes Cost-effectiveness Group (2002) Cost-effectiveness of intensive glycemic control, intensified hypertension control, and serum cholesterol level reduction for type 2 diabetes. JAMA 287: 2542-2551.

35. Diabetes Prevention Program Research Group1 (2012) The 10-year costeffectiveness of lifestyle intervention or metformin for diabetes prevention: an intent-to-treat analysis of the DPP/DPPOS. Diabetes Care 35: 723-730.

36. Herman WH, Edelstein SL, Ratner RE, Montez MG, Ackermann RT, et al (2013) Diabetes Prevention Program Research Group. Effectiveness and costeffectiveness of diabetes prevention among adherent participants. Am J Manag Care 19:194-202

37. Diabetes Prevention Program Research Group (2003) Within-trial costeffectiveness of lifestyle intervention or metformin for the primary prevention of type 2 diabetes. Diabetes Care 26: 2518-2523.

38. Eddy DM1, Schlessinger L (2003) Archimedes: a trial-validated model of diabetes. Diabetes Care 26: 3093-3101.

39. Mason JM, Freemantle N, Gibson JM, New JP (2005) SPLINT trial. Specialist nurse-led clinics to improve control of hypertension and hyperlipidemia in diabetes: economic analysis of the SPLINT trial. Diabetes Care 28:40-46

40. New JP, Mason JM, Freemantle N, Teasdale S, Wong LM, et al. (2003) Specialist nurse-led intervention to treat and control hypertension and hyperlipidemia in diabetes (SPLINT): a randomized controlled trial. Diabetes Care 26: 2250-2255.

41. Gillett M, Dallosso HM, Dixon S, Brennan A, Carey ME, et al. (2010) Delivering the diabetes education and self-management for ongoing and newly diagnosed (DESMOND) programme for people with newly diagnosed type 2 diabetes: cost effectiveness analysis. BMJ 341:c4093

42. Davies MJ, Heller S, Skinner TC, Campbell MJ, Carey ME, et al. (2008) Diabetes Education and Self-Management for Ongoing and Newly Diagnosed
Collaborative. Effectiveness of the diabetes education and self-management for ongoing and newly diagnosed (DESMOND) programme for people with newly diagnosed type 2 diabetes: cluster randomised controlled trial. BMJ 336:491-495

43. Dijkstra RF, Niessen LW, Braspenning JC, Adang E, Grol RT (2006) Patientcentred and professional-directed implementation strategies for diabetes guidelines: a cluster-randomized trial-based cost-effectiveness analysis. Diabe Med 23:164-170.

44. Dijkstra RF, Braspenning JC, Huijsmans Z, Akkermans RP, van Ballegooie $E$, et al. (2005) Introduction of diabetes passports involving both patients and professionals to improve hospital outpatient diabetes care. Diabetes Res Clin Pract 68: 126-134.

45. Graves N, Barnett AG, Halton KA, Veerman JL, Winkler E, et al (2009) Costeffectiveness of a telephone-delivered intervention for physical activity and diet PLoS One 4: e7135

46. Eakin E, Reeves M, Lawler S, Graves N, Oldenburg B, et al. (2009) Telephone counseling for physical activity and diet in primary care patients. Am J Prev Med 36: 142-149.

47. Cleveringa FG, Welsing PM, van den Donk M, Gorter KJ, Niessen LW, et al. (2010) Cost-effectiveness of the diabetes care protocol, a multifaceted computerized decision support diabetes management intervention that reduces cardiovascular risk. Diabetes Care 33:258-263

48. Cleveringa FG, Gorter KJ, van den Donk M, Rutten GE (2008) Combined task delegation, computerized decision support, and feedback improve cardiovascular risk for type 2 diabetic patients: a cluster randomized trial in primary care. Diabetes Care 31: 2273-2275.

49. Eastman RC, Javitt JC, Herman WH, Dasbach EJ, Copley-Merriman C, et al (1997) Model of complications of NIDDM. II. Analysis of the health benefits and cost-effectiveness of treating NIDDM with the goal of normoglycemia. Diabetes Care 20: 735-744.

50. Handley MA, Shumway M, Schillinger D (2008) Cost-effectiveness of automated telephone self-management support with nurse care management among patients with diabetes. Ann Fam Med 6: 512-518.

51. Schillinger D, Hammer H, Wang F, Palacios J, McLean I, et al. (2008) Seeing in 3-D: examining the reach of diabetes self-management support strategies in a public health care system. Health Educ Behav 35: 664-682.

52. Mason JM, Freemantle N, Gibson JM, New JP; SPLINT trial (2005) Specialist nurse-led clinics to improve control of hypertension and hyperlipidemia in diabetes: economic analysis of the SPLINT trial. Diabetes Care 28: 40-46.

53. Young RJ, Taylor J, Friede T, Hollis S, Mason JM, et al. (2005) Pro-active call center treatment support (PACCTS) to improve glucose control in type 2 diabetes: a randomized controlled trial. Diabetes Care 28:278-282.

54. Irvine L, Barton GR, Gasper AV, Murray N, Clark A, et al. (2011) Costeffectiveness of a lifestyle intervention in preventing Type 2 diabetes. Int $\mathrm{J}$ Technol Assess Health Care 27: 275-282.

55. Simon J, Gray A, Clarke P, Wade A, Neil A, et al. (2008) Cost effectiveness of self monitoring of blood glucose in patients with non-insulin treated type 2 diabetes: economic evaluation of data from the DiGEM trial. BMJ 336: 11771180.

56. Farmer A, Wade A, Goyder E, Yudkin P, French D, et al. (2007) Impact of selfmonitoring of blood glucose in the management of patients with non-insulin treated diabetes: open parallel group randomised trial. BMJ 335:132

57. Cleemput I, Neyt M, Thiry N, De Laet C, Leys M (2008) Threshold values for cost-effectiveness in health care Health Technology Assessment (HTA) Brussels: Belgian Health Care Knowledge Centre (KCE) KCE reports $100 \mathrm{C}$ (D/2008/10.273/96)

58. Waugh N, Scotland G, McNamee P, Gillett M, Brennan A, et al. (2007) Screening for type 2 diabetes: literature review and economic modelling. Health Techno Assess 11: iii-iv, ix-xi, 1-125.

Citation: Odnoletkova I, Goderis G, Pil L, Nobels F, Aertgeerts B, et al. (2014) Cost-Effectiveness of Therapeutic Education to Prevent the Development and Progression of Type 2 Diabetes: Systematic Review. J Diabetes Metab 5: 438 doi:10.4172/2155-6156.1000438 